\title{
Effects of Alisma Decoction on lipid metabolism and inflammatory response are mediated through the activation of the LXR $\alpha$ pathway in macrophage-derived foam cells
}

\author{
XIEHUA XUE ${ }^{1}$, TONG CHEN ${ }^{2}$, WEI WEI ${ }^{1}$, XIAOMAO ZHOU ${ }^{2}$, ZHICHENG LIN $^{1}$ and LIDIAN CHEN ${ }^{2}$ \\ ${ }^{1}$ Affiliated Rehabilitation Hospital, Fujian University of Traditional Chinese Medicine, ${ }^{2}$ College of Rehabilitation Medicine, \\ Fujian University of Traditional Chinese Medicine, Fuzhou, Fujian 350003, P.R. China
}

Received December 7, 2013; Accepted January 31, 2014

DOI: $10.3892 /$ ijmm.2014.1646

\begin{abstract}
The liver X receptor $\alpha(\mathrm{LXR} \alpha) / \mathrm{ATP}$-binding cassette transporter A1 (ABCA1) pathway and LXR-modulated cytokines play an important role in macrophages which mediate lipid engulfment and the inflammatory response, and participate in the process of atherosclerosis. Therefore, lipid-lowering and anti-inflammatory therapy through the activation of the LXR $\alpha / A B C A 1$ pathway and LXR $\alpha$-modulated cytokines may prove to be one of the main treatment strategies for atherosclerosis. Alisma Decoction (AD) has long been used in China to clinically treat cardiovascular and cerebral diseases; however, the precise mechanisms involved remain to be elucidated. In the present study, we evaluated the regulation of lipids and the anti-inflammatory effects exerted by $\mathrm{AD}$ and investigated the underlying molecular mechanisms using oxidized low-density lipoprotein (ox-LDL)-stimulated foam cells derived from rat peritoneal macrophages. We first found that AD markedly relieved lipid deposition in foam cells as it increased LXR $\alpha$ and ABCA1 expression and decreased the ox-LDL-induced expression of inflammatory cytokines, such as matrix metalloproteinase-9 and interleukin-1 $\beta$. Collectively, our findings suggest that blocking lipid deposition and inhibiting inflammatory response through the activation of the LXR $\alpha$ pathway may be one of the main mechanisms through which AD exerts its anti-atherosclerotic effects.
\end{abstract}

Correspondence to: Professor Lidian Chen, College of Rehabilitation Medicine, Fujian University of Traditional Chinese Medicine, 282 WuSi Road, Gulou, Fuzhou, Fujian 350003, P.R. China

E-mail: cld@fjtcm.edu.cn

Abbreviations: AS, atherosclerosis; AD, Alisma Decoction; RPMs, rat peritoneal macrophages; ox-LDL, oxidized low-density lipoprotein; Dil-ox-LDL, Dil-labeled oxidized low-density lipoprotein; ABCA1, ATP-binding cassette transporter $\mathrm{A} 1 ; \mathrm{LXR} \alpha$, liver $\mathrm{X}$ receptor $\alpha$; MMP-9, matrix metalloproteinase-9; IL-1 $\beta$, interleukin-1 $\beta$

Key words: traditional Chinese medicine, Alisma Decoction, foam cell, liver X receptor- $\alpha$, ATP-binding cassette transporter A1, matrix metalloproteinase-9, interleukin-1 $\beta$

\section{Introduction}

Atherosclerosis (AS) is a type of disease in which lipid metabolism disorder and chronic inflammation co-exist. Macrophages also play an important role in this type of disease. The development of AS is closely associated with the engulfment of oxidized low-density lipoprotein (ox-LDL) by macrophages. Macrophages engulf ox-LDL unrestrictedly through the scavenger receptor, resulting in a large number of lipids being deposited, further promoting the formation of foam cells, and the secretion of large amounts of enzyme matrix metalloproteinases (MMPs) and a variety of cytokines, which play an important role in the occurrence and development of AS (1). Liver X receptors (LXRs) are members of the nuclear receptor (NR) superfamily of ligand-activated transcription factors and exist as two isoforms, LXR $\alpha$ and LXR $\beta$ (2). LXRs are intracellular lipid sensors that are activated by oxysterols in response to elevated intracellular cholesterol levels in multiple cell types (3). Within macrophages, LXRs protect the cells against foam cell formation through the activation of reverse cholesterol transport (4). Studies have demonstrated that several genes involved in the regulation of the balance of lipid metabolism, inflammation and immune response are regulated by LXRs (5). LXR forms a heterodimer with the retinoid X receptor; together they can bind to a variation of a consensus sequence (DR4)TGACCGNNNNTAACCT in the promoter of the ATP-binding cassette transporter A1 (ABCA1), as well as other genes, inducing an upregulated expression (6). LXR then regulates cholesterol efflux and maintains the balance of lipid metabolism. Activated LXRs can also suppress the expression of pro-inflammatory cytokines, such as MMP-9

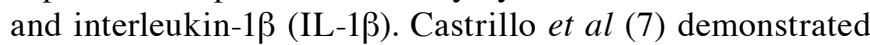
that the activation of LXRs inhibited the expression of MMP-9 in macrophages, at least in part through the antagonism of the NF- $\mathrm{KB}$ signaling pathway. Therefore, LXRs play an important role in anti-atherosclerosis by regulating lipid metabolism and inflammatory mediators, and have became one of the hotspots in drug development in recent years $(8,9)$.

Alisma Decoction (AD) is a classical traditional Chinese formula that was first prescribed in the Eastern Han Dynasty, which consists of a combination of two herbs, including alisma and atractylodes. AD has long been used in China in the treat- 
ment of cardiovascular and cerebral diseases (10). Certain studies have demonstrated the lipid-lowering, anti-atherosclerotic and other pharmacological effects of AD $(11,12)$. However, the mechanisms responsible for these effects have not yet been fully elucidated. In the present study, using the most important cells involved in the development of AS, macrophage-derived foam cells, we investigated the effects of $\mathrm{AD}$ on lipid metabolism and inflammatory responses in foam cells, as well as the mechanisms involved.

\section{Materials and methods}

Materials and reagents. DMEM-F12 was purchased from HyClone Laboratories (Shanghai, China). Fetal bovine serum (FBS) was from Gibco-BRL (Gaithersburg, MD, USA). ox-LDL $(1.26 \mathrm{mg} / \mathrm{l})$ and Dil-labeled oxidized low-density lipoprotein (Dil-ox-LDL) $(1.9 \mathrm{mg} / \mathrm{ml})$ were obtained from Beijing Xie Sheng Ssheng Wu Company (Beijing, China); both Oil Red $\mathrm{O}$ and hematoxylin were obtained from Wuhan Boster Biological Technology Ltd., Wuhan, China; 3-(4,5-dimethylthiazol-2-yl)-5-(3-carboxymethoxyphenyl)-2-(4-sulfophenyl)2H-tetrazolium (MTS) was obtained from Promega Corp. (Madison, WI, USA). Antibodies used in western blot analysis included the following: i) anti-ABCA1 antibody (AB.H10) mouse monoclonal antibody; ii) anti-LXR $\alpha$ antibody (PPZ0412) (mouse monoclonal antibody; iii) MMP-9 antibody (ab76003) rabbit monoclonal (EP1254); iv) (all from Abcam, Shanghai, China). Anti- $\beta$-actin (BM0627) mouse; v) and horseradish peroxidase (HRP)-conjugated secondary antibodies were purchased from Wuhan Boster Biological Technology Ltd.. The cytokine IL-1 $\beta$ ELISA kit was purchased from IBL Systems Inc., Hamburg, Germany. ECL reagents and cell lysates were acquired from the Beyotime Institute of Biotechnology, Jiangsu, China. Male Sprague-Dawley (SD) rats were provided by the Experimental Animal Center of Fujian Medical University, Fuzhou, China [License no., SCXK (Min) 2012-0001; the western blotting electrophoresis equipment was obtained from Bio-Rad, Shanghai, China; the cell incubator was purchased from Thermo Fisher Scientific Inc., Waltham, MA, USA. The Leica DM IL LED inverted microscope was from Leica Instrument Co., Ltd., Beijing, China.

Preparation of water extract from $A D$. Crude materials of $\mathrm{AD}$ were supplied by the Fujian University of Traditional Chinese Medicine Rehabilitation Hospital Pharmacy and were carefully identified. Alisma and atractylodes were soaked in water for $30 \mathrm{~min}$ and mixed in proportion and decocted twice by refluxing with water $(1: 6$ and then $1: 4, \mathrm{w} / \mathrm{v})$ for $1 \mathrm{~h}$. The filtrates were combined and condensed. They were then stored at $4^{\circ} \mathrm{C}$ until use.

Preparation of $A D$-containing serum. A total of 40 male SD rats (weighing 200-220 g) were used in this study. The rats underwent a period of adaptive for 3 days before being used in the experiments. The rats were then randomly divided into the $\mathrm{AD}$-containing serum group and the control group (same amount of saline injection; $n=20$ in each group). The rats were administered the treatments $(1 \mathrm{ml} / 100 \mathrm{~g})$ by gavage twice a day for 7 days. Abdominal aortic blood was then collected after gavage for $2 \mathrm{~h}$ on the eighth day. Subsequently, blood serum was separated by centrifugation at $3,000 \mathrm{rpm}$ at $4^{\circ} \mathrm{C}$ for $10 \mathrm{~min}$. The serum was then mixed, inactivated for $30 \mathrm{~min}$ at $56^{\circ} \mathrm{C}$, and the bacteria were filtered through a microporous membrane, and stored at $-20^{\circ} \mathrm{C}$ until use. All procedures associated with the care of the animals were performed according to the National Institutes of Health Guide for the Care and Use of Laboratory Animals and were approved by the Institutional Animal Care and Use Committee of Fujian University of Traditional Chinese Medicine.

Cytotoxity assay. The rat peritoneal macrophages (RPMs) were grown in 96-well plates and then incubated with $\mathrm{AD}$-containing serum at various concentrations (10, 20 and $30 \%$ ) for 12,24 and $48 \mathrm{~h}$. MTS assay was used to measure the viability of the cells. Cells were incubated at $37^{\circ} \mathrm{C}$ with MTS $(1.90 \mathrm{mg} / \mathrm{ml})$ for $4 \mathrm{~h}$. The resulting color was assayed at $490 \mathrm{~nm}$ using a microplate absorbance reader (Thermo Fisher Scientific Inc.).

RPM extracts, cell culture and grouping. RPMs were collected by lavaging the peritoneal cavity with $10 \mathrm{ml}$ of DMEM-F12. Then the rats were soaked in $75 \%$ ethanol for $5 \mathrm{~min}$. Cells were collected by centrifugation $\left(1,000 \times \mathrm{g}, 15 \mathrm{~min}, 4^{\circ} \mathrm{C}\right)$ and suspended in DMEM-F12 supplemented with 10\% FBS. For the experiments, the cells were subcultured in 6 -well or $25 \mathrm{~cm}^{2}$ tissue culture dishes at a density of $5 \times 10^{6}$ cells/well and maintained in a humidified incubator with $5 \% \mathrm{CO}_{2}$ at $37^{\circ} \mathrm{C}$. The RPMs were randomly assigned to four groups: i) the control group, in which the cells were incubated in DMEM-F12 supplemented with $10 \%$ FBS for $24 \mathrm{~h}$; ii) the ox-LDL group, where the cells were incubated in DMEM-F12 containing $10 \%$ FBS with oxidized LDL (50 mg/l) or Dil-ox-LDL $(10 \mu \mathrm{g} / \mathrm{ml})$ for $24 \mathrm{~h}$; iii) the ox-LDL + AD group, where the foam cells were incubated in DMEM-F12 containing 10\% FBS with AD-containing serum and oxidized LDL $(50 \mathrm{mg} / \mathrm{l})$ or Dil-ox-LDL $(10 \mu \mathrm{g} / \mathrm{ml})$ for $24 \mathrm{~h}$; and iv) the AD group, where the cells were treated with the AD-containing serum for $24 \mathrm{~h}$.

Morphological observation. After removal of the culture medium, the cells were washed three times with phosphatebuffered saline (PBS) and then fixed for 15 min with 5\% formalin solution. Thereafter, the cells were stained with Oil Red O for $10 \mathrm{~min}$, followed by hematoxylin staining for $5 \mathrm{~min}$. The cells were then observed under a microscope and images were acquired at $\mathrm{x} 40$ magnification.

The RPMs were cultured in DMEM-F12 with $10 \%$ FBS for $24 \mathrm{~h}$ and treated with or without the $10 \mu \mathrm{g} / \mathrm{ml}$ DIL-ox-LDL for an additional $24 \mathrm{~h}$. The culture medium was then removed. The cells were washed and harvested with PBS. Coverslips were mounted and analyzed under a fluorescent microscope.

Western blot analysis. Whole cell protein extracts were prepared from the RPMs. The cells were washed with PBS and lysed by adding lysis buffer. After mixing at $4^{\circ} \mathrm{C}$ for $20 \mathrm{~min}$, the samples were centrifuged at $12,000 \mathrm{xg}$ for $10 \mathrm{~min}$ at $4^{\circ} \mathrm{C}$, and the supernatant was collected and stored at $-80^{\circ} \mathrm{C}$. Protein assays were performed using the Bradford assay (Bio-Rad). Proteins $(30 \mu \mathrm{g})$ were separated on an 10\% SDS-polyacrylamide gel and transferred onto PVDF membranes with a $0.45-\mu \mathrm{m}$ pore size (IPVH00010; Millipore, Billerica, MA, USA) for $1.5 \mathrm{~h}$ 
at $110 \mathrm{~V}$ at $4^{\circ} \mathrm{C}$. After electro-transfer, the membranes were incubated in Blotto (5\% non-fat dry milk in TBS) for $1 \mathrm{~h}$ at room temperature. The membranes were then washed three times, $10 \mathrm{~min}$ each, in TBS-Tween-20 at room temperature. They were then incubated with polyclonal antibody against LXR $\alpha$ or ABCA1 or MMP-9 overnight. The membranes were washed with TBS-Tween-20 as described above, and then primary antibodies were detected with goat anti-rabbit-IgG or rabbit anti-mouse-IgG conjugated to horseradish peroxidase, diluted 1:3,000 in Blotto for $2 \mathrm{~h}$ at room temperature. Subsequently, the membranes were washed and peroxidase activity was detected by enhanced chemiluminescence.

Measurement of $I L-1 \beta$. The macrophages were plated in a 6 -well cell culture plate and treated with the various treatments as described above (RPM extracts, cell culture and grouping). Following the manufacturer's instructions, a volume of $1 \mathrm{ml}$ of culture-medium supernatant was collected for measurement of the concentration levels of IL-1 $\beta$ by the relevant ELISA kit.

Statistical Analysis. Three or more separate experiments were performed. The results are expressed as a the means \pm standard error (SE), and the data were analyzed using one-way ANOVA followed by Dunnett's test or Student's t-test using SigmaPlot (SigmaPlot for Windows, version 16.0; Systat Software Inc., San Jose, CA, USA) to determine any significant differences. Values of $\mathrm{P}<0.05$ or $\mathrm{P}<0.01$ were considered to indicate statistically significant differences.

\section{Results}

MTS assay. We performed MTS assay to evaluate the effects of AD-containing serum on cell viability. AD-containing serum did not display any cytotoxic effects in the RPMs. Following treatment with various concentrations of $\mathrm{AD}$-containing serum, the cells had a better viability at the concentration of $20 \%$ (Fig. 1).

Oil Red $O$ staining. The RPMs were treated with ox-LDL (50 mg/l) for $24 \mathrm{~h}$, then washed twice with PBS, and stained with Oil Red $\mathrm{O}$ and hematoxylin. There were a large number of red lipid droplets in the cell cytoplasm in the ox-LDL group and nuclei were blue by hematoxylin staining. Thus, the foam cell model derived from RPMs was successfully established. However, following treatment with $20 \%$ AD-containing serum, the intracellular lipid droplets became lighter in color (lighter red) and lipid deposition was alleviated (Fig. 2).

Immunofluorescence. The RPMs were treated with Dil-ox-LDL $(10 \mu \mathrm{g} / \mathrm{ml})$ for $24 \mathrm{~h}$. The foam cells were then observed under a fluorescence microscope. Dil-ox-LDL engulfed by RPMs showed red fluorescence. However, following therapeutic intervention with $20 \%$ AD-containing serum, the Dil-ox-LDL fluorescence showed weaker staining in the foam cells. This suggested that lipid deposition was attenuated in the foam cells following treatment with AD-containing serum (Fig. 3).

Protein expression of ABCAI and LXR in macrophagederived foam cells folloiwng treatment with AD-containing serum. To examine the effects of AD-containing serum on

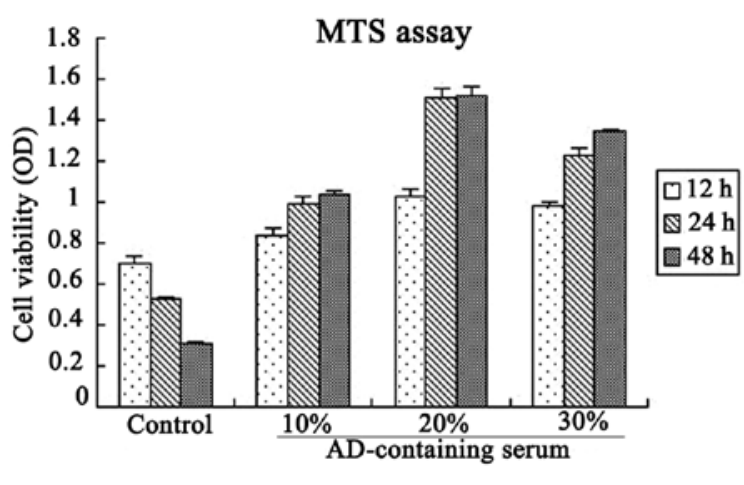

Figure 1. Effect of Alisma Decoction (AD)-containing serum on rat peritoneal macrophage cell viability. Cells were treated with various concentrations of AD-containing serum for 12, 24 and $48 \mathrm{~h}$. Cell viability was evaluated by MTS assay. Optical density (OD) reflects the viability of the cells. Each value indicates the mean \pm standard deviation (SD) from at least three independent experiments.

the expression of LXR $\alpha$ and ABCA1, following the specified treatments for 12, 24 and $48 \mathrm{~h}$, the cells were collected, washed twice with ice-cold PBS, and were then subjected to protien extraction and cell lysis, and then protein levels were quantified. Western blot analysis was utilized to detect the protein expression of LXR $\alpha$ and ABCA1. The results revealed no significant difference in LXR $\alpha$ expression between the control and ox-LDL group; the expression level of LXR $\alpha$ was low in the control group and ox-LDL group. This suggested that ox-LDL (50 mg/l) marginally effected LXR $\alpha$ levels. However, the ox-LDL + AD and AD group had the highest expression level of $\operatorname{LXR} \alpha$. Thus, the present data reveal that AD activates LXR $\alpha$ in normal macrophages and foam cells (Fig. 4). Compared with the control group, the ABCA1 protein expression level was significantly increased in the ox-LDL, ox-LDL + AD and AD groups (Fig 5). These results raise the possibility that ox-LDL $(50 \mathrm{mg} / \mathrm{l})$, as well as AD-containing serum, can upregulate the expression of ABCA1 in foam cells. Although the ox-LDL + AD group showed a slightly decreased expression of ABCA1 compared with ox-LDL group, no statistical significance was observed. Thus, these data suggest that AD-containing serum increases ABCA1 protein expression by activating $\mathrm{LXR} \alpha$.

Effect of AD-containing serum on MMP-9 expression in macrophage-derived foam cells. MMP-9 protein expression was detected by western blot analysis. MMP-9 protein expression in the ox-LDL group was markedly increased compared with the control group (Fig. 6). However, following treatment with $20 \%$ AD-containing serum, the protein level markedly decreased in a time-dependent manner compared with the ox-LDL group. However, when comparing the cells in the control group with those treated with ox-LDL alone at $48 \mathrm{~h}$, no statistical significance was observed.

Measurement of IL-1 $\beta$ expression by ELISA. For the determination of IL- $1 \beta$ production in the extracellular medium, culture supernatants were collected to determine IL-1 $\beta$ activity. Treatment of the macrophages with $50 \mathrm{mg} / \mathrm{l}$ ox-LDL resulted in a marked increase in the secretion of IL-1 $\beta$. However, 


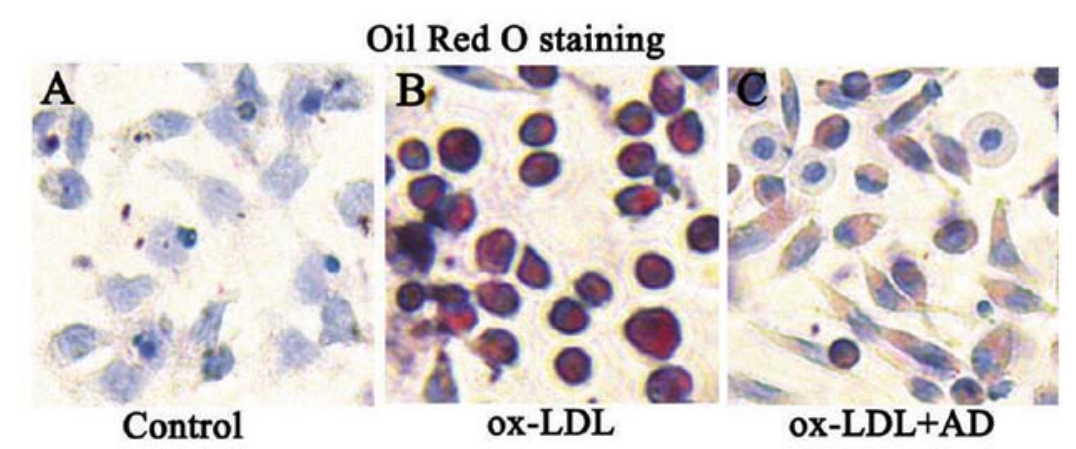

Figure 2. Oil Red O staining: transformation of macrophages to foam cells and the changes in intracellular lipid levels following treatment with oxidized lowdensity lipoprotein (ox-LDL) and/or Alisma Decoction (AD)-containing serum. Images were captured under a microscope at a magnification of $x 400$. Images are representative of three independent experiments. (A) The macrophages not treated with ox-LDL or AD were visualized under a microscope, and the cells were counterstained with hematoxylin (blue). (B) The macrophages were observed following stimulation with ox-LDL, and the cells engulfed a large number of lipids (red), stained by Oil Red O. (C) The macrophages were detected follwoing treatment with AD, and lipids in the cell cytoplasm markedly decreased.

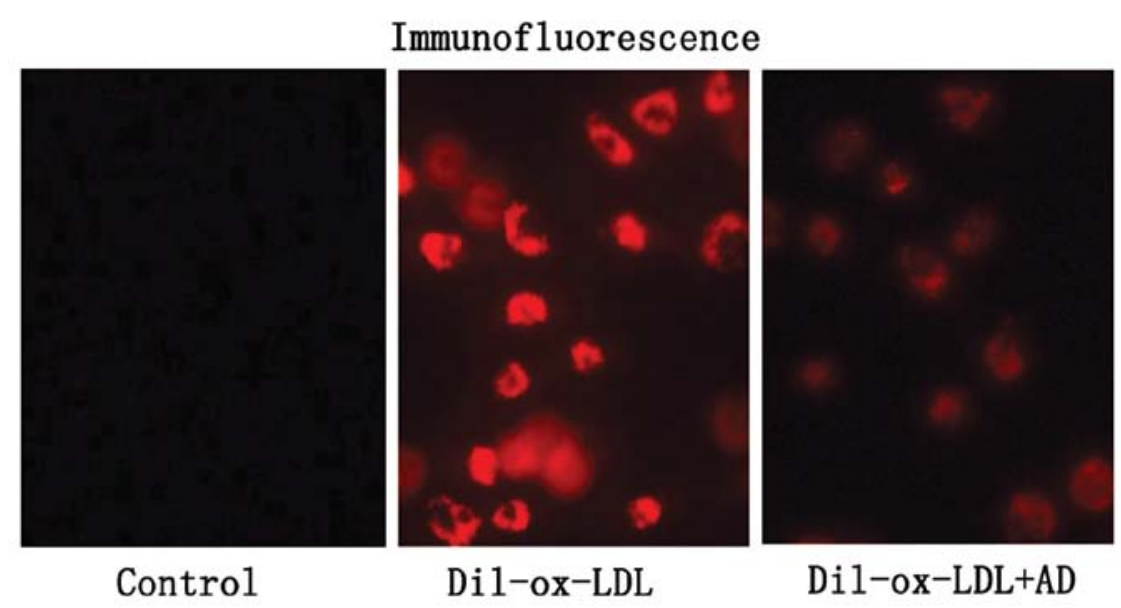

Figure 3. Changes in lipid Dil-labeled oxidized low-density lipoprotein (Dil-ox-LDL) deposition following the different treatments, as observed under a fluorescence microscope. Images were captured under a confocal fluorescence microscope with a magnification of $x 400$. Images are representative of three independent experiments. The macrophages in the control group were not detected, as these cells did not show red fluorescence. In the Dil-ox-LDL group, a high amount of lipids was found deposited in the cells, emitting red fluorescence. In the Dil-ox-LDL + AD group, the lipid deposition of the cells was markedly reduced, as shown by the decrease in red fluorescence. AD, Alisma Decoction.

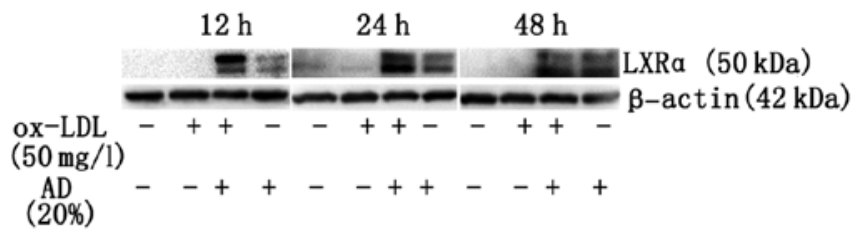

Figure 4. Effect of Alisma Decoction (AD) treatment on liver $\mathrm{X}$ receptor $\alpha$ $(\mathrm{LXR} \alpha)$ protein expression in foam cells derived from rat peritoneal macrophages. Cells were treated with AD for 12,24 and $48 \mathrm{~h}$. The protein expression of LXR $\alpha$ was determined by western blot analysis. $\beta$-actin was used as the internal control. Images are representatives of three independent experiments.

following treatment with $\mathrm{AD}$-containing serum, IL-1 $\beta$ expression significantly decreased $(\mathrm{P}<0.05)$, as determined by ELISA. Moreover, the most significant reduction in IL-1 $\beta$ expression was observed following treatment with $\mathrm{AD}$-containing serum for $24 \mathrm{~h}$, demonstrating the anti-inflammatory effects of $\mathrm{AD}$ in macrophages (Fig. 7). However, there was no statistically significant differene observed at 12 and $48 \mathrm{~h}$.

\section{Discussion}

AS is a complex vascular disorder. Both hyperlipidemia and inflammation are well known risk factors for the development of AS and have been shown to play a causal role in the progression of atherosclerotic plaques (13). Macrophages play a crucial role in atherogenesis, through the engulfment of modified LDL and the secretion of inflammatory modulators, cytokines and matrix-degrading enzymes (14). The accumulation of ox-LDL in atherosclerotic lesions is a principal event in atherosclerosis. ox-LDL not only enhances atherogenesis but also drives arterial inflammation, thus promoting the progression of AS (15).

Being important modulators of the macrophage cholesterol efflux and inducers of anti-inflammatory responses, LXR activation results in the amelioration of the plasma lipoprotein profile by mobilizing reverse cholesterol transport $(4,16)$. LXRs also appear to be important modulators of inflammation through the direct regulation of inflammatory gene expression. Hong and Tontonoz (17), as well as others demonstrated that the 

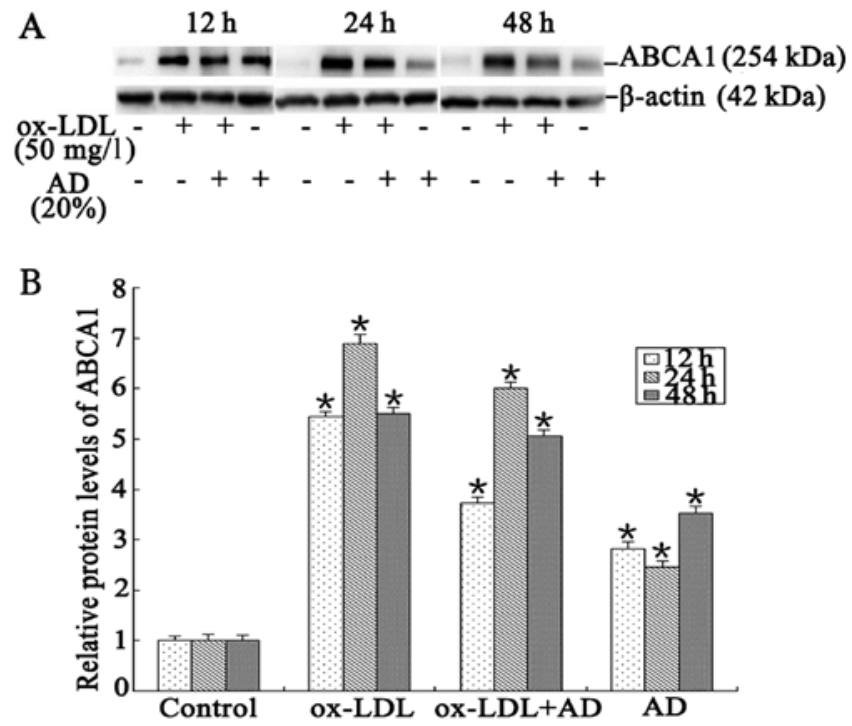

Figure 5. Effect of Alisma Decoction (AD) treatment on ATP-binding cassette transporter A1 (ABCA1) protein expression in foam cells derived from rat peritoneal macrophages. Cells were treated with $\mathrm{AD}$ for 12,24 and $48 \mathrm{~h}$. (A) The protein expression of ABCA1 was determined by western blot analysis. $\beta$-actin was used as the internal control. Images are representative of three independent experiments. (B) Statistical analysis: ${ }^{*} \mathrm{P}<0.05$ represents the three treatment groups compared with the control group.
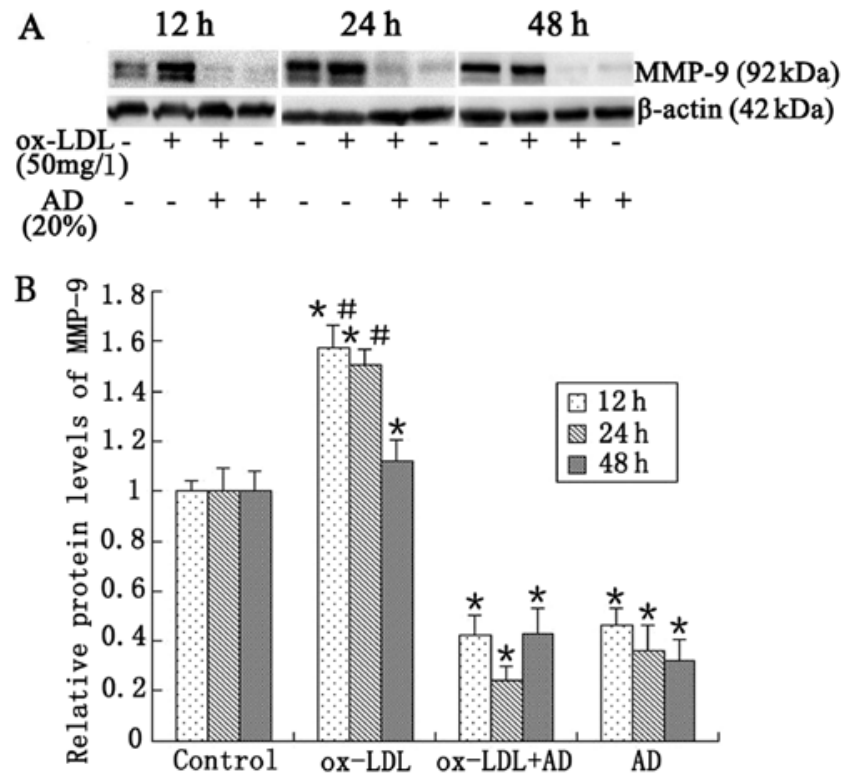

Figure 6. Effect of Alisma Decoction (AD) treatment on matrix metalloproteinase-9 (MMP-9) protein expression in foam cells derived from rat peritoneal macrophages. Cells were treated with $\mathrm{AD}$ for 12,24 and $48 \mathrm{~h}$. (A) The protein expression of MMP-9 was determined by western blot analysis. $\beta$-actin was used as the internal control. Images are representative of three independent experiments. (B) Statistical analysis: ${ }^{*} \mathrm{P}<0.05$ represents the three intervention groups compared with the control cells, and cells treated with oxidized low-density lipoprotein (ox-LDL) $(50 \mathrm{mg} / \mathrm{l})$ and AD (20\%) compared with cells treated with ox-LDL alone at $48 \mathrm{~h}$; ${ }^{~} \mathrm{P}<0.01$ represents cells treated with ox-LDL and AD compared with the cells treated with ox-LDL alone at 12 and $24 \mathrm{~h}$.

activation of LXRs inhibits the induction of pro-inflammatory gene expression following LPS stimulation or bacterial infec-

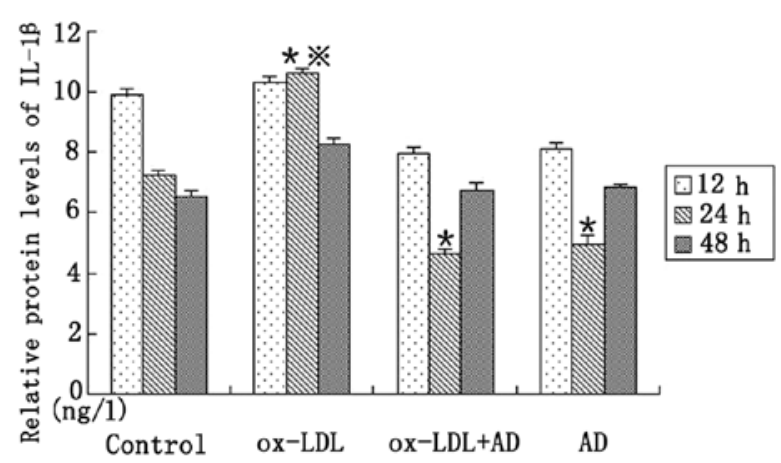

Figure 7. Effect of different treatments on IL-1 $\beta$ protein expression in foam cells derived from rat peritoneal macrophages. Cells were treated with various treatments for 12, 24 and $48 \mathrm{~h}$. Alisma Decoction (AD) represents AD-containing serum in this figure. (1) The protein expression of IL-1 $\beta$ was determined by ELISA. (2) Statistical analysis: ${ }^{*} \mathrm{P}<0.05$ represents the three intervention groups compared with the untreated control cells at $24 \mathrm{~h} ;{ }^{*} \mathrm{P}<0.05$ represents cells treated with oxidized low-density lipoprotein (ox-LDL) $(50 \mathrm{mg} / \mathrm{l})$ and $\mathrm{AD}(20 \%)$ compared with the cells treated with ox-LDL alone at $24 \mathrm{~h}$.

tion (18). LXRs inhibit inflammatory cytokines, including iNOS, COX2, IL-6 and IL-1 $\beta$ and MMP-9 (19).

MMPs play important roles in acute and chronic inflammation, partly by promoting the turnover of the extracellular matrix (ECM) and partly by modulating the actions of inflammatory mediators (20). MMP-9 is one of the main MMPs involved in the degradation of the ECM and type IV collagen (21). MMP-9 expression significantly increases and has been linked with inflammation, diabetic microvascular complications, ECM degradation and synthesis, and cardiac dysfunction (22). There is only a small amount of MMP-9 expression in the normal arterial wall; however, in atherosclerotic plaques, the expression of a variety of MMPs can be detected, of which MMP-9 has a stronger activity (23). Macrophages are an important cellular source of plasma MMP-9 (24). Therefore, the inhibition of MMP-9 expression in macrophages may have anti-atherosclerotic effects, leading to plaque stabilization. It has been previously demonstrated that MMPs can be activated by oxidative stress (25). Huang et al (26) reported that the activation of MMPs occurs through intermediates of ox-LDL. In parallel with this result, in the present study, we found that MMP-9 expression markedly increased followign treatment with ox-LDL. By contrast, AD-containing serum effectively reduced the expression of MMP-9 in a time-dependent manner in macrophage-derived foam cells.

In addition, it is worth noting that macrophages are also the major source of IL-1 $\beta$ as the predominant inflammatory-type cells in atherosclerotic lesions $(27,28)$.IL-1 $\beta$ is considered to be a prototypic 'multifunctional' cytokine in inflammation $(29,30)$. Previous studies have shown that IL-1 $\beta$ has a significasnt effect on the initiation and progression of AS $(31,32)$. The cytokines produced by macrophages in atherosclerotic lesions are regarded to be important in the initiation and amplification of IL-1 $\beta$-mediated inflammation (33). Macrophages are induced by ox-LDL to secrete IL-1 $\beta$ (34). In line with this report, our study demonstrated that IL-1 $\beta$ expression markedly increased following treatment with ox-LDL. However, IL-1 $\beta$ expression 
significantly decreased in the cells treated with AD-containing serum, particularly at $24 \mathrm{~h}$ after stimulation.

Futhermore, dyslipidemia and inflammation are well known and equally important risk factors for the development of atherosclerosis. The interplay between lipid metabolism and inflammation at multiple levels in metabolic active tissues may exacerbate the development of atherosclerosis. It is worth noting that ABCA1 is among the earliest identified target genes for LXR, and whose activation has been shown to promote reverse cholesterol transport in vivo (35). Chawla et al (36) reported that ox-LDL upregulated the expression of ABCA1 through the activation of the peroxisome proliferator-activated receptor- $\gamma$-LXR pathway in macrophages. In parallel with these results, we found that the ox-LDL + AD group and AD group showed a higher protein expression level of LXR $\alpha$ and ABCA1 compared with the control group; thus, we can speculate that $\mathrm{AD}$ attenuated lipid deposition by regulating $\mathrm{LXR} \alpha$ and ABCA1 in macrophages. However, when the ox-LDL + AD group and AD group were compared with the ox-LDL group, the former showed a higher expression level of LXR $\alpha$, but it did not appear that ABCA1 expression had a higher level similar to LXR $\alpha$. On the contrary, the expression level of ABCA1 decreased slightly. We consider that the reasons for this may be the following: certain studies have suggested that ABCA1 not only plays the role of transporter on cellular cholesterol and phospholipids, but it also increases the secretion of inflammatory cytokines, such as IL-1 $\beta$ and monocyte chemoattractant protein-1 (MCP-1) at the cellular level (37,38). Yin et al (39) reported that inflammatory cytokines, such as IL- $1 \beta$ and interferon (IFN)- $\gamma$ inhibit ABCA1 expression; Reddy et al (40) found that ABCA1 is not only involved in reverse cholesterol transport, but that it also participates in the oxidative modification of LDL. ABCA1 plays an important role in the oxidative modification of LDL by regulating the release of active oxygen on the arterial wall. The inhibition of ABCAl expression can prevent LDL-induced lipid peroxidation. Thus, we believe that ox-LDL can directly activate the expression of ABCA1, while the high expression of ABCA1 promotes the oxidative modification of LDL. Accordingly, it can be inferred that ABCA1 may play a dual role in the process of AS. The dynamic balance between the expression and degradation of ABCA1 in vivo plays an important role in maintaining the metabolic balance of lipids. In addition, studies have shown that the half-life of ABCA1 protein is only $1-2 \mathrm{~h}$, and it is always in the process of homeostasis between expression and degradation (41). Therefore, it can be hypothesized that ABCA1 protein expression is not only effected by LXR $\alpha$, ox-LDL and its short half-life, by is also influenced by various inflammatory cytokines secreted from macrophages and lipid oxidation, maintaining a dynamic equilibrium state. This partly explains this seemingly contradictory phenomenon. Thus, it is suggested that AD markedly blocked lipid deposition in macrophages by activating the LXR $\alpha$ ABCA1 signaling pathway. However, ABCA1 is affected by secreting inflammatory cytokines from macrophages, as well as metabolism itself; thus, ABCA1 protein expression remains at a suitable equilibrium, rather than increasing unlimitedly. Hence, the effects of and regulation of ABCA1 expression by pathologic stimuli remain controversial.

In conclusion, this study demonstrates that Alisma Decoction reduces lipid deposition of RPM-derived foam cells and inhibits the secretion of MMP-9 and IL-1 $\beta$ through the activation of LXR $\alpha$. Taken together, these results suggest a potential role for Alisma Decoction in the protection against and treatment of AS.

\section{Acknowledgements}

This study was sponsored by grants from the National Natural Science Foundation of China (no. 81001543/H2717) and the Key Project of the Department of Health of Fujian Province (no. Wzzk0905).

\section{References}

1. Lusis AJ: Atherosclerosis. Nature 407: 233-241, 2000.

2. Bonamassa B and Moschetta A: Atherosclerosis: lessons from LXR and the intestine. Trends Endocrinol Metab 24: 120-128, 2013.

3. van Diepen JA, Berbée JF, Havekes LM and Rensen PC: Interactions between inflammation and lipid metabolism: Relevance for efficacy of anti-inflammatory drugs in the treatment of atherosclerosis. Atherosclerosis 228: 306-315, 2013.

4. Venkateswaran A, Laffitte BA, Joseph SB, et al: Control of cellular cholesterol efflux by the nuclear oxysterol receptor LXR alpha. Proc Natl Acad Sci USA 97: 12097-12102, 2000.

5. Zelcer $\mathrm{N}$ and Tontonoz $\mathrm{P}$ : Liver $\mathrm{X}$ receptors as integrators of metabolic and inflammatory signaling. J Clin Invest 116: 607-614, 2006.

6. Edwards PA, Kennedy MA and Mak PA: LXRs; oxysterolactivated nuclear receptors that regulate genes controlling lipid homeostasis. Vascul Pharmacol 38: 249-256, 2002.

7. Castrillo A, Joseph SB, Marathe C, et al: Liver X receptordependent repression of matrix metalloproteinase- 9 expression in macrophages. J Biol Chem 278: 10443-10449, 2003.

8. Giannarelli C, Cimmino G, Connolly TM, et al: Synergistic effect of liver $\mathrm{X}$ receptor activation and simvastatin on plaque regression and stabilization: an magnetic resonance imaging study in a model of advanced atheroselerosis. Eur Heart J 33: 264-273, 2012.

9. Calkin AC and Tontonoz P: Liver x receptor signaling pathways and atherosclerosis. Arterioscler Thromb Vasc Biol 30: 1513-1518, 2010.

10. Liv J: The efficacy evaluation of Alisma Decoction assiting on western medicine treating essential hypertension. Shi Yong Yao Wu Yu Lin Chuang 16: 629-630, 2013 (In Chinese).

11. Liv S, Cao K and Wang P: Alisma Decoction treating hyperlipidemia by 120 Cases. Zhong Yi Yao Lin Chuang Za Zhi 17: 454, 2005 (In Chinese).

12. Tang X, Zhai Y and Liu T: The effect of Alisma Decoction on hemorheology and serum nitric oxide on experimental hyperlipidemia rats. Zhong Guo Shi Yan Fang Ji Xue Za Zhi 12: 26-28, 2006 (In Chinese).

13. Steinberg D: Atherogenesis in perspective: hypercholesterolemia and inflammation as partners in crime. Nat Med 8: 1211-1217, 2002.

14. Glass CK and Witztum JL: Atherosclerosis. The road ahead. Cell 104: 503-516, 2001.

15. Jiang Y, Wang M, Huang $\mathrm{K}$, et al: Oxidized low-density lipoprotein induces secretion of interleukin-1 $\beta$ by macrophages via reactive oxygen species-dependent NLRP3 inflammasome activation. Biochem Biophys Res Commun 425: 121-126, 2012.

16. Repa JJ, Turley SD, Lobaccaro JA, et al: Regulation of absorption and ABC1-mediated efflux of cholesterol by RXR heterodimers. Science 289: 1524-1529, 2000.

17. Hong $\mathrm{C}$ and Tontonoz $\mathrm{P}$ : Coordination of inflammation and metabolism by PPAR and LXR nuclear receptors. Curr Opin Genet Dev 18: 461-467, 2008.

18. Joseph SB, Castrillo A, Laffitte BA, Mangelsdorf DJ and Tontonoz P: Reciprocal regulation of inflammation and lipid metabolism by liver X receptors. Nat Med 9: 213-219, 2003.

19. Qian Y and Ni Z: The effect of liver X receptors in metabolism and inflammation. Lin Chuang Xin Xue Guan Bing Za Zhi 23: 806-810, 2007 (In Chinese).

20. Parks WC and Shapiro SD: Matrix metalloproteinases in lung biology. Respir Res 2: 10-19, 2001.

21. Ferretti G, Bacchetti T, Marotti E and Curatola G: Effect of homocysteinylation on human high-density lipoproteins: a correlation with paraoxonase activity. Metabolism 52: 146-151, 2003. 
22. Fang L, Du XJ, Gao XM and Dart AM: Activation of peripheral blood mononuclear cells and extracellular matrix and inflammatory gene pro file in acute myocardial infarction. Clin Sci (Lond) 119: 175-183, 2010.

23. Halade GV, Jin YF and Lindsey ML: Matrix metalloproteinase (MMP)-9: a proximal biomarker for cardiac remodeling and a distal biomarker for inflammation. Pharmacol Ther 139: 32-40, 2013.

24. Krex D, Schachert HK and Schackert G: Genesis of cerebral aneurysms - an update. Acta Neurochir (Wien) 143: 429-449, 2001.

25. Sigala F, Kotsinas A, Savari P, et al: Oxidized LDL in human carotid plaques is related to symptomatic carotid disease and lesion instability. J Vasc Surg 52: 704-713, 2010.

26. Huang Y, Mironova M and Lopes-Virella MF: Oxidized LDL stimulates matrix metalloproteinase-1 expression in human vascular endothelial cells. Arterioscler Thromb Vasc Biol 19: 2640-2647, 1999.

27. Moyer CF, Sajuthi D, Tulli H and Williams JK: Synthesis of IL-1 alpha and IL-1 beta by arterial cells in atherosclerosis. Am J Pathol 138: 951-960, 1991.

28. Tipping PG and Hancock WW: Production of tumor necrosis factor and interleukin-1 by macrophages from human atheromatous plaques. Am J Pathol 142: 1721-1728, 1993.

29. Church LD, Cook GP and McDermott MF: Primer: inflammasomes and interleukin 1beta in inflammatory disorders. Nat Clin Pract Rheumatol 4: 34-42, 2008.

30. Dinarello CA: Anti-inflammatory agents: present and future. Cell 140: 935-950, 2010.

31. Rader DJ: IL-1 and atherosclerosis: a murine twist to an evolving human story. J Clin Invest 122: 27-30, 2012.

32. Kirii H, Niwa T and Yamada Y: Lack of interleukin-1beta decreases the severity of atherosclerosis in ApoE-deficient mice, Arterioscler Thromb Vasc Biol 23: 656-660, 2003.
33. Dinarello CA: Biologic basis for interleukin-1 in disease. Blood 87: 2095-2147, 1996.

34. Ku G, Thomas CE, Akeson AL and Jackson RL: Induction of interleukin 1beta expression from human peripheral blood monocyte-derived macrophages by 9 -hydroxyoctadecadienoic acid. J Biol Chem 267: 14183-14188, 1992.

35. Naik SU, Wang X, Da Silva JS, et al: Pharmacological activation of liver $\mathrm{X}$ receptors promotes reverse cholesterol transport in vivo. Circulation 113: 90-97, 2006.

36. Chawla A, Boisvert WA, Lee CH, et al: A PPAR gamma-LXRABCA1 pathway in macrophages is involved in cholesterol efflux and atherogenesis. Mol Cell 7: 161-171, 2001.

37. Guo ZG, Wu PS, Li JH and Lai WY: Modulation of inflammatory cytokines by ATP-binding cassette A1 in THP-1 macrophages. Nan Fang Yi Ke Da Xue Xue Bao 26: 1269-1271, 2006 (In Chinese).

38. Li J, Yang Y, Hao P and Guo Z: ABCA1 regulating on inflammatory cytokines and its significance on vascular smooth muscle cells. Shi Yong Yi Xue Za Zhi 26: 1713-1716, 2010 (In Chinese).

39. Yin K, Liao DF and Tang CK: ATP-binding membrane cassette transporter A1 (ABCA1): a possible link between inflammation and reverse cholesterol transport. Mol Med 16: 438-449, 2010.

40. Reddy ST, Hama S, Ng C, Grijalva V, Navab $M$ and Fogelman AM: ATP-binding cassette transporter-1 participates in LDL Oxidation by artery wall cells. Arterioscler Thromb Vasc Biol 22: 1877-1883, 2002.

41. Zhou X, Yin Z, Guo X, Hajjar DP and Han J: Inhibition of ERK1/2 and activation of liver $X$ receptor synergistically induce macrophage ABCA1 expression and cholesterol efflux. J Biol Chem 285: 6316-6326, 2010. 